DOI: https://doi.org/10.46296/gt.v1i1.0001

\title{
MAMOPLASTIA DE AUMENTO: ABORDAJE FISIOTERAPÉUTICO
}

\section{MAMOPLASTIA DE AUMENTO: ABORDAJE FISIOTERAPÉUTICO}

\author{
Fernández-Palma Claudia ${ }^{1 *}$ \\ ${ }^{1}$ Investigadora independiente. Manta, Ecuador.
}

*Correo: claufer-pal@gmail.com

\begin{abstract}
Resumen
En la actualidad, el número de cirugías estéticas realizadas en el país sigue en aumento, específicamente aquellas que van encaminadas en el mejoramiento de la apariencia de los senos por su relación con la belleza en la cultura latina; cada vez este tipo de intervención se está llevando a cabo en personas de edades tempranas, casi cercanas a la adolescencia. El objetivo de esta investigación es dar a conocer la importancia del abordaje e intervención fisioterapéutica, partiendo de las principales complicaciones generadas por la realización de la cirugía estética de mamoplastia de aumento en la fase postoperatoria, siendo en este caso la contractura capsular. Para ello se llevó a cabo la revisión de diversos artículos científicos de carácter internacional y de diferentes bases de datos con el fin de cumplir el objetivo propuesto en la investigación. Asimismo, se denotó que mientras más temprana sea la intervención por parte del fisioterapeuta a través del drenaje linfático manual, la aplicación de ultrasonido y la inducción miofascial, existen mayores posibilidades de reducir las complicaciones relacionadas con la cirugía estética e incorporación del paciente a sus actividades cotidianas.
\end{abstract}

Palabras clave: Cirugía plástica, mamoplastia de aumento, fisioterapia dermatofuncional, contractura capsular, intervención fisioterapéutica.

\begin{abstract}
Currently, the number of cosmetic surgeries performed in the country continues to increase, specifically those that are aimed at improving the appearance of the breasts due to their relationship with beauty in Latin culture; each time this type of intervention is being carried out in people of early ages, almost close to adolescence. The objective of this research is to make known the importance of the physiotherapeutic approach and intervention, based on the main complications generated by performing augmentation mammoplasty cosmetic surgery in the postoperative phase, in this case being capsular contracture. For this, a review of various scientific articles of an international nature and of different databases was carried out in order to fulfill the objective proposed in the research. Likewise, it was noted that the earlier the intervention by the physiotherapist through manual lymphatic drainage, the application of ultrasound and myofascial induction, there are greater possibilities of reducing complications related to cosmetic surgery and incorporation of the patient into their activities. everyday.
\end{abstract}

Keywords: Plastic surgery, augmentation mammoplasty, dermatofunctional physiotherapy, capsular contracture, physiotherapeutic intervention.

Información del manuscrito:

Fecha de recepción: 26 de octubre de 2017

Fecha de aceptación: 15 de diciembre de 2017

Fecha de publicación: 10 de enero de 2018 


\section{Introducción}

La fisioterapia es la ciencia del área de la salud que tiene como principal objeto de estudio el movimiento corporal humano, dicha área profesional en los últimos años ha experimentado una fuerte demanda en las diversas casas de estudios que existen en nuestro país.

Además de contar con un abordaje en el área cardiorrespiratoria, pediátrica, neurológica, deportiva, ortopédica, también tiene participación en el área estética o mejor conocida como fisioterapia dermatofuncional, la cual es la responsable del abordaje del sistema tegumentario como un todo, incluyendo las alteraciones de la piel.

En este caso particular, el objetivo del presente manuscrito es difundir el abordaje desde el punto de vista de la fisioterapia, las principales complicaciones de la mamoplastia de aumento, las técnicas más efectivas en el tratamiento postoperatorio según sus efectos fisiológicos. Del mismo modo, se presenta el equipo interdisciplinario idóneo para realizar este tipo de cirugías y las recomendaciones desde el punto de vista fisioterapéutico antes de realizar una cirugía de esta envergadura.

\section{Mamoplastia de aumento}

\subsection{Cirugía}

Según Duque (2011) la mamoplastia de aumento se define como un procedimiento mediante el cual se aumenta el tamaño de los senos, a través de la introducción de un implante o prótesis. Esta cirugía se lleva a cabo mayormente por un índice de satisfacción de la percepción corporal y una mejora de la autoestima en la mayoría de los casos. Además, ocupa el primer lugar entre las cirugías estéticas en Estados Unidos, según la American Society of Plastic Surgeons, alrededor de 280.000 mujeres se sometieron a una cirugía estética de mamoplastia de aumento en el año 2009

Existen varios tipos de implantes, los cuales van desde gel de silicona líquido, gel de silicona cohesivo, o implante de suero salino, hasta diferentes localizaciones del mismo, ya sea por debajo del pectoral mayor conocida como subpectoral, la cual es la más utilizada y la que ofrece mejores resultados desde el punto de vista estético. Sin embargo, también se encuentra la localización 
subglandular, misma que consiste en alojar el implante entre la glándula mamaria y la musculatura de la zona.

Las prótesis mamarias de silicona provocan el desarrollo de una envoltura fibrosa 0 cápsula periprotésica. La contractura de la cápsula, por retracción del tejido fibroso, es la complicación más frecuente e importante de estos implantes, puesto que produce un endurecimiento de grado variable $y$, en los casos avanzados, deformidad de la mama. Se ha relacionado estrechamente con el tipo de superficie del implante (lisa 0 texturada) y con la disposición de las fibras de colágeno, habiéndose sugerido que la formación de una cápsula continua, con fibras de colágeno dispuestas paralela y circularmente alrededor de las prótesis lisas, promueve el desarrollo de fuerzas contráctiles concéntricas, que traccionando al unísono producirían la contractura.

Con las prótesis texturadas microporosas se ha demostrado una incidencia significativamente más baja de contractura capsular en comparación con las lisas. Se considera que el crecimiento tisular dentro de la estructura microporosa origina fuerzas contráctiles multidireccionales, con tendencia a neutralizarse entre ellas cuando su efecto se suma sobre el implante.

La cápsula de estos implantes presenta una zona basal compuesta de múltiples capas de fibras de colágeno alineadas paralelamente, las cuales podrían contraerse. Sin embargo, la adherencia de las prótesis texturadas microporosas podría contrarrestar las fuerzas contráctiles.

Los motivos que incitan a la realización de mamoplastia van desde la reducción del tejido mamario 0 el aumento, hasta reconstrucción producto de accidentes y padecimiento de cáncer de mamas. Asimismo, se cuenta con la participación de un equipo interdisciplinario antes y después de la programación de una cirugía de este tipo.

El abordaje fisioterapéutico no siempre es recomendado por los médicos cirujanos en la fase preoperatoria, así lo demuestra el artículo científico sobre Análise descritiva do encaminhamento médico a tratamentos fisioterapêuticos dermato-funcionais nos períodos pré e pós-operatório de 
cirugías plásticas cosméticas, realizado en la ciudad de Porto Alegre, en Brasil, con una población de 30 médicos cirujanos, denotando que el $13 \%$ de los participantes admitieron desconocer la fisioterapia dermatofuncional en períodos pre y postoperatorios y un $93 \%$ indicó que solo la recomienda en período postoperatorio. En cuanto al equipo responsable del procedimiAento quirúrgico lo conforma el médico cirujano, anestesiólogo y demás ayudantes para llevar a cabo la cirugía.

\subsection{Complicaciones}

Las complicaciones que surgen luego de este tipo de cirugía plástica vienen dadas por tres factores principales: depende del lugar donde se realice la incisión, el plano de inserción y el tipo de implante que se utilice, como lo menciona Lesavoy et al. (2010).

La complicación más común es la contractura capsular con el 5,5\%, esta suele ocupar el primer lugar según un estudio realizado por Tebbetts (2006), seguido de pliegues y contornos irregulares (4,7\%), deflación del implante (3,8\%), cambios transitorios sensoriales en el brazo (3,5\%), colección localizada del líquido axilar (2,3\%), reintervención por mal posicionamiento del implante $(2 \%)$ y por último hematomas $(0,2 \%)$. Este estudio fue realizado a un total de 690 pacientes, de los cuales 66 tenían ubicado el implante en el plano submamario, 322 en el plano subpectoral y 282 con doble plano.

La contractura capsular se origina cuando la cápsula, que normalmente se encuentra presente alrededor del implante se torna gruesa y comprime la prótesis mamaria, causando la deformación de la misma y generando una manifestación tanto de dolor como de malestar psicológico por la apariencia física de los senos.

La etiología suele ser muy variable según Escudero et al., (2005), puesto que la causa se puede vincular con un exceso de miofibroblastos, ya que los mismos son necesarios para la contracción de las heridas y de modo que si estos no están presentes en gran cantidad se impide la cicatrización de manera efectiva. En el mismo estudio se demuestra que el tejido capsular encontrado es sensible a algunos fármacos que buscan relajar o estimular el músculo liso. 
Otra de las complicaciones que produce este tipo de cirugía estética es el efecto a largo plazo, que se presenta en el aparato locomotor, esto se debe al peso extra generado por los implantes mamarios, lo que hace insuficiente la capacidad muscular del sujeto y obliga a generar cambios posturales viciosos, que conllevan a modificaciones estructurales principalmente en la columna vertebral.

\section{Abordaje fisioterapéutico}

La recuperación debe iniciar dos o tres días luego de la intervención y debe ser lo más cómoda posible y rápida a fines de que la misma se concrete con éxito en la recuperación del paciente, garantizando así los resultados deseados.

El principal objetivo luego de la realización de la mamoplastia será la disminución del edema ocasionado a través de la reabsorción de la linfa, la cual es un líquido de color claro que se acumula en toda la zona de las mamas y crea la sensación de dolor.

La linfa es reabsorbida por los vasos linfáticos ubicados en todo el cuerpo $y$ se les denomina capilares linfáticos, cabe señalar que estos desembocan en los precolectores que son los vasos que transportan la linfa, y estos últimos desembocan en los colectores (Leduc \& Leduc, 2006).

\section{Drenaje linfático manual}

El drenaje linfático manual no tiene una definición consensuada, no obstante, se trata de una técnica de masaje que tiene como objetivo mejorar la reabsorción del edema. En este sentido, Ferrandez (2006) concluye que el fisioterapeuta que practica el drenaje linfático manual tiene como propósito favorecer dos fases de la fisiología linfática en su papel circulatorio, la captación a través de las variaciones de presiones realizadas en la zona edematizada, con unos cambios generados en el gradiente de presión hidrostática entre el espacio intersticial y el capilar linfático que crean la penetración en la luz linfática del líquido intersticial y los elementos que la componen.

Por otro lado, la evacuación se aplica a lo largo de los ejes de los colectores superficiales, estimulando su contracción mediante el estiramiento de los linfangiones. 


\section{Otros métodos}

Además, la Revista Aesthetic Plast Surg en el 2006 presentó un método de analgesia mediante la aplicación de infiltraciones con toxina botulínica en el pectoral mayor y el serrato anterior, proporcionando la disminución del dolor y mejorando la amplitud del movimiento por el efecto que tiene la misma en las estructuras microscópicas del músculo.

Por otro lado, la aplicación del ultrasonido se considera beneficiosa, debido a que genera la transferencia de ondas mecánicas de mayor frecuencia que el sonido, estas ondas crean un movimiento celular que genera un efecto térmico, provocando un mayor aporte vascular y oxigenación en el tejido, que es favorable en el proceso de cicatrización.

Sin embargo, el incremento de la flexibilidad de los tejidos ricos en colágeno, la estimulación de las funciones celulares y de regeneración tisular también forman parte de los efectos fisiológicos provocados por este agente físico.

Asimismo, se presentan otras técnicas como la inducción Miofascial. Esta última se dirige a trabajar específicamente sobre la fascia, que es el tejido conectivo que recubre los músculos, huesos, articulaciones, vísceras, y está conformada por estructuras nerviosas, vasculares, contráctiles y además es rica en colágeno. La fascia es la responsable de mantener la estructura del cuerpo unida.

Mediante la aplicación de esta técnica se pretende aprovechar las propiedades coloidales del colágeno para influir en la forma de la fascia y así eliminar las restricciones que no permiten al colágeno presentar su verdadera estructura.

\section{Conclusiones}

La importancia de conocer como se lleva a cabo el procedimiento de la mamoplastia de aumento, el tipo de materiales utilizados por los médicos cirujanos y la localización de los implantes, permite tener una mejor perspectiva del procedimiento para realizar un abordaje óptimo desde la fisioterapia.

Si bien es cierto que todavía se desconoce el rol del fisioterapeuta a grandes rasgos y solo se reconoce su participación en la fase postoperatoria, se deben realizar 
esfuerzos para transformar esa percepción a través de la intervención que se brinda a los pacientes. Por tanto, es indispensable considerar la participación desde la etapa preoperatoria, donde el enfoque de la misma se proyecta en aras de preparar todo el sistema musculoesquelético respecto al cambio que se generará, con el fin de evitar cambios estructurales, que comprometan la funcionabilidad del individuo.

Como se ha expresado en el desarrollo del presente artículo la complicación más frecuente que aqueja a los que se realizan estos procedimientos quirúrgicos es sin duda la contractura capsular, además, se ha podido demostrar que para la misma existen varias opciones terapéuticas que permiten evitar una posible afección en la calidad del movimiento de la zona cercana a la intervención.

En este sentido, es el drenaje linfático manual la técnica ideal para la disminución de la inflamación y reabsorción del edema provocado. No obstante, la aplicación del ultrasonido por su incremento del metabolismo local, junto con la participación de funciones celulares y la capacidad de regeneración tisular han demostrado ser beneficiosas para el proceso de cicatrización, tan importante en la prevención de la formación de contractura capsular. La innovación de la infiltración con toxina botulínica como método analgésico, permite además favorecer la amplitud de movimiento.

Y por último la utilización de la inducción miofascial, como una herramienta de tratamiento global que busca un resultado múltiple a través de la eliminación de restricciones en la fascia.

Siguiendo el mismo orden de ideas, es importante crear el reconocimiento de la labor del fisioterapeuta dentro de ámbito de la estética, y la manera más oportuna para lograrlo apunta a la investigación, generando evidencia científica que sustente los conocimientos teórico-prácticos. Este paso debe comenzar por quienes ya se han atrevido a incursionar en la fisioterapia dermatofuncional, creando así el aporte más valioso para las generaciones de relevo. 


\section{Bibliografía}

Duque, M. (2011) ¿Qué tipos de prótesis hay disponibles? Revista Sociedad colombiana de cirugía plástica. Colombia.

Escudero, F. J., Guarch, R., \& Lozano, J. A. (2005). Reacción tisular a las prótesis mamarias: Contractura capsular periprotésica. In Anales del Sistema Sanitario de Navarra (Vol. 28, pp. 4153). Gobierno de Navarra. Departamento de Salud.

Esposito, G., Gravante, G., Marianetti, M., \& Delogu, D. (2006). "Reverse" dual-plane mammaplasty. Aesthetic plastic surgery, 30(5), 521526.

https://doi.org/10.1007/s0026 6-006-0066-z

Ferrandez, J. (2006) El sistema linfático historia, iconografía e implicaciones

fisioterapéuticas. Madrid. Ed Panamericana.

Leduc, A., \& Leuduc, O. (2006) Drenaje linfático teoría y práctica. $\quad 1^{\circ}$ Edición. Barcelona. Ed Masson.
Lesavoy, M. A., Trussler, A. P., \& Dickinson, B. P. (2010). Difficulties with subpectoral augmentation mammaplasty and its correction: the role of subglandular site change in revision aesthetic breast surgery. Plastic and reconstructive surgery, 125(1), 363-371.

Milani, G., João, S. M., \& Farah, E. A. (2006). Fundamentos da Fisioterapia dermatofuncional: revisão de literatura. Fisioterapia $E$ Pesquisa, 13(1), 37-43. https://doi.org/10.1590/fpusp. v13i1.76159

Tebbetts, J. B. (2006). Axillary endoscopic breast augmentation: processes derived from a 28-year experience to optimize outcomes. Plastic and reconstructive surgery, 118(7S), 53S-80S. $10.1097 / 01$.prs.0000247314. 92351.99 